\title{
Targeting tumor-associated fibroblasts improves cancer chemotherapy by increasing intratumoral drug uptake
}

\author{
Markus Loeffler, ${ }^{1}$ Jörg A. Krüger, ${ }^{1}$ Andreas G. Niethammer, ${ }^{2}$ and Ralph A. Reisfeld ${ }^{1}$ \\ ${ }^{1}$ The Scripps Research Institute, Department of Immunology, La Jolla, California, USA. ${ }^{2}$ Department of Radiation Oncology, \\ Heidelberg Medical School, Heidelberg, Germany.
}

\begin{abstract}
Tumor-associated fibroblasts are key regulators of tumorigenesis. In contrast to tumor cells, which are genetically unstable and mutate frequently, the presence of genetically more stable fibroblasts in the tumor-stromal compartment makes them an optimal target for cancer immunotherapy. These cells are also the primary source of collagen type $I$, which contributes to decreased chemotherapeutic drug uptake in tumors and plays a significant role in regulating tumor sensitivity to a variety of chemotherapies. To specifically kill tumor-associated fibroblasts, we constructed an oral DNA vaccine targeting fibroblast activation protein (FAP), which is specifically overexpressed by fibroblasts in the tumor stroma. Through $\mathrm{CD8}^{+} \mathrm{T}$ cell-mediated killing of tumor-associated fibroblasts, our vaccine successfully suppressed primary tumor cell growth and metastasis of multidrug-resistant murine colon and breast carcinoma. Furthermore, tumor tissue of FAP-vaccinated mice revealed markedly decreased collagen type I expression and up to $70 \%$ greater uptake of chemotherapeutic drugs. Most importantly, pFap-vaccinated mice treated with chemotherapy showed a 3-fold prolongation in lifespan and marked suppression of tumor growth, with $50 \%$ of the animals completely rejecting a tumor cell challenge. This strategy opens a new venue for the combination of immuno- and chemotherapies.
\end{abstract}

\section{Introduction}

It has become increasingly clear that tumor antigens are elusive targets for cancer therapy (1). This can be explained by a variety of characteristics unique to tumor cells, including mutations of their antigens and inadequate antigen presentation to $\mathrm{T}$ cells due to MHC class I antigen downregulation (2-4). Moreover, defects in apoptosis signaling pathways as well as upregulation of apoptosis inhibitors such as survivin, XIAP, or antiapoptotic members of the bcl-2 family (5) confer resistance not only to T cell-mediated killing but also to the apoptosis-inducing effect of chemotherapeutic agents. In order to overcome these obstacles, genetically more stable cells in the tumor stroma may prove to be a promising cellular target for cancer immunotherapy.

There is growing recognition that the stromal compartment plays a crucial role in tumorigenesis and invasion (6-8). Stromal cells have been shown to stimulate the transformation of normal epithelial cells, and to produce growth factors, cytokines, and chemokines that activate the adjacent ECM and induce the selection and expansion of neoplastic cells $(6,7)$. Earlier studies have demonstrated that tumor cells inoculated in suspension were not tumorigenic whereas fragments of solid tumors containing their stroma led to tumor growth (9). This activated ECM also conferred chemoresistance mediated by $\beta_{1}$-integrins that adhere to fibronectin, leading to the activation of $\beta_{1}$-integrin-stimulated tyrosine kinase, which suppresses chemotherapy-induced apoptosis. This was shown in doxorubicin-sensitive myeloma cells, which developed resistance after adherence to fibronectin (8). In fact, recent reports have indicated that tumor rejection can be achieved by

Nonstandard abbreviations used: FAP, fibroblast activation protein; $\mathrm{pFap}$, pcDNA3.1/V5-His-TOPO-Fap.

Conflict of interest: The authors have declared that no conflict of interest exists. Citation for this article: J. Clin. Invest. 116:1955-1962 (2006). doi:10.1172/JCI26532. modulation of tumor-stromal fibroblasts (10) or by disturbance of the tumor-stromal network $(11,12)$. In addition, it has been demonstrated that tumor-associated macrophages and fibroblasts synthesize proteins, such as VEGF, TGF- $\beta_{1}$, and IL-10, which contribute to the local immunosuppressive environment and render tumor cells more chemoresistant (13-17).

Fibroblasts in the tumor stroma synthesize fibroblast activation protein (FAP), a type II transmembrane protein that functions as a serine protease. FAP is selectively overexpressed in over $90 \%$ of stromal fibroblasts associated with colon, breast, and lung carcinomas (18). Transient overexpression of FAP can also be found during wound healing and in some fetal mesenchymal tissues. Additionally, this enzyme was reported to cleave gelatin and collagen type I (19) and has therefore been implicated in ECM remodeling. Overexpression of FAP reportedly leads to promotion of tumor growth and increases in metastatic potential, whereas treatment with anti-FAP antibodies or pulsed dendritic cells inhibits tumor growth $(20,21)$. Furthermore, tumor-stromal expression and organization of collagen type I, which is mainly produced by fibroblasts, is inversely correlated with intratumoral uptake of various molecules, such as IgG (22), BSA (23), or chemotherapeutic agents (24); this substantiates the involvement of the stromal compartment in resistance to chemotherapy. Fibroblasts in the tumor stroma also show signs of differentiation toward smooth muscle with contractile properties that may contribute to an increased interstitial fluid pressure (25).

Taken together, these findings suggest that the tumor-stromal antigen FAP can serve as a novel target for active vaccination against cancer, especially if combined with chemotherapy. We explored this possibility by demonstrating that an effective $\mathrm{CD} 8^{+} \mathrm{T}$ cell-mediated anti-FAP immune response could be induced that significantly suppressed primary tumors as well as established metastatic tumor growth of multidrug-resistant murine CT26 colon and D2F2 breast 
A

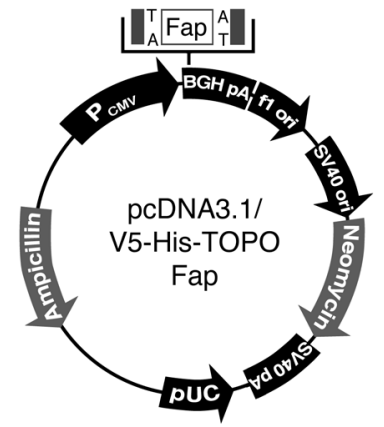

B
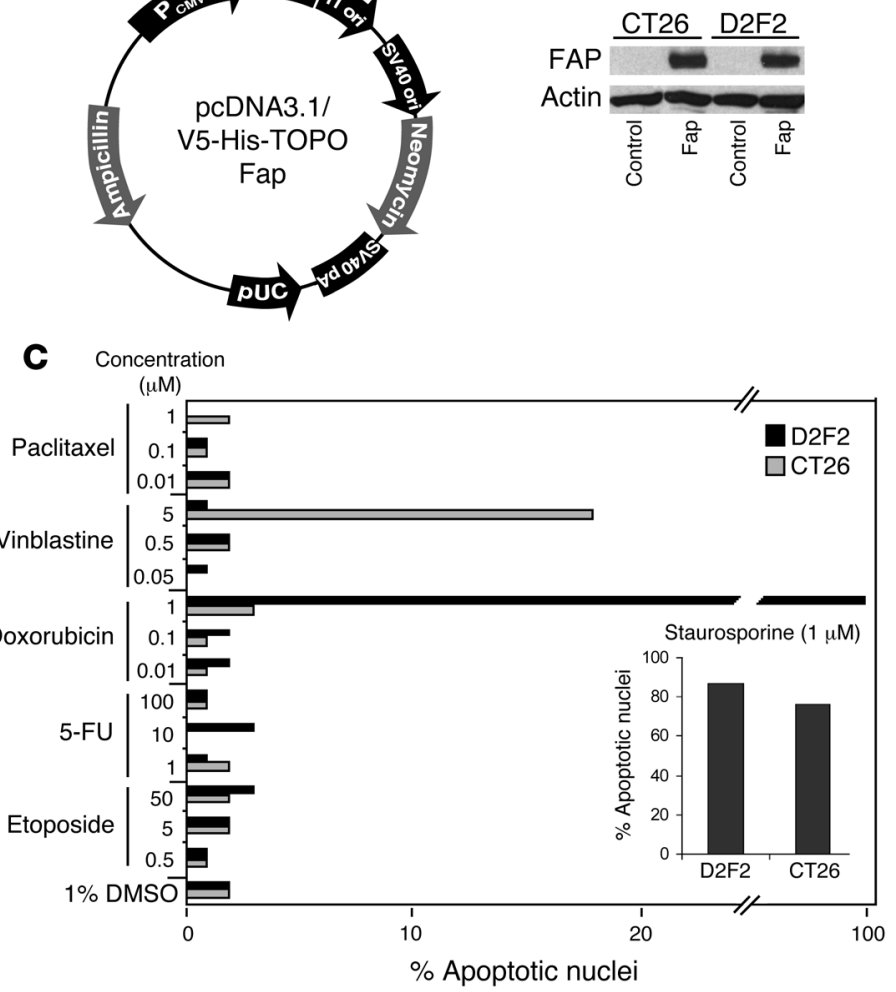

\section{Figure 1}

Characterization of the FAP construct and chemoresistant tumor cell lines. (A) The cDNA encoding the entire murine FAP, verified by nucleotide sequencing, was inserted into the EcoRI site of the pFap vector. (B) Protein expression was demonstrated by Western blotting after transient transfection of CT26 and D2F2 cells.

(C) CT26 colon and D2F2 breast carcinoma cells were treated with various chemotherapeutic agents at the concentrations indicated. After 48 hours of incubation, nuclear apoptosis was assessed by staining with Hoechst 33342 dye. Staurosporine was used as a positive control. $\mathrm{P}_{\mathrm{CMV}}$, human cytomegalovirus immediate-early promoter/enhancer; BGHpA, bovine growth hormone polyadenylation signal; f1 ori, f1 origin; SV40 ori, SV40 early promoter and origin; neomycin, neomycin (G418) resistance gene; SV40pa, SV40 polyadenylation signal; pUC, pUC-derived origin; ampicillin, ampicillin resistance gene ( $\beta$-lactamase). carcinoma cells. More importantly, we were able to demonstrate that interference with tumor-associated fibroblasts can serve as a novel strategy to decrease intratumoral collagen type I and thereby increase uptake of chemotherapeutic agents. Combination of chemotherapy with the FAP vaccine resulted in a marked decrease of primary breast tumor growth, with $50 \%$ of the animals completely rejecting the challenge and a 3 -fold prolongation of survival in an experimental breast cancer metastasis model.

\section{Results}

Construction and expression of the vector pcDNA3.1/V5-His-TOPO-Fap. After constructing the eukaryotic expression vector pcDNA3.1/ V5-His-TOPO-Fap (pFap) as described in Methods (Figure 1A), we demonstrated the correct expression of the $88-\mathrm{kDa}$ FAP protein by Western blotting of cell lysates from both transiently transfected CT26 colon carcinoma and D2F2 breast carcinoma cells, which themselves do not express FAP (Figure 1B).

Multidrug resistance of CT26 and D2F2 cell clones. After using the CT26 colon cancer and D2F2 breast cancer cell lines in our laboratory for several years, we found that clones of both cell lines had acquired chemoresistance. Upon addition of 5 apoptosis-inducing chemotherapeutic agents (Figure 1C), solely vinblastine was able to induce minor apoptotic effects in the CT26 colon carcinoma clone, although only at the very high concentration of $5 \mu \mathrm{M}$. It has previously been reported that the $\mathrm{IC}_{50}$ for 5 -fluorouracil of the original CT26 cell line is $1.85 \mu \mathrm{M}(22)$. However, we were unable to induce nuclear apoptosis in our clone even at a concentration as high as $100 \mu \mathrm{M}$. In D2F2 breast carcinoma cells, only doxorubicin, tested at the highest concentration of $1 \mu \mathrm{M}$, was able to induce nuclear apoptosis as determined by staining with Hoechst 33342 dye. We thus concluded that both cell lines had acquired multidrug resistance during the prolonged handling in our laboratory.

Prophylactic treatment with the FAP-based DNA vaccine in hibits primary tumor growth. Ten days after the last of 3 immunizations by gavage at 1-week intervals, we challenged different groups of BALB/c mice $(n=8)$ either s.c. with CT26 colon carcinoma cells or orthotopically with D2F2 breast carcinoma cells as described in Methods. We were able to show that an orally administrated DNA vaccine encoding murine FAP could indeed suppress primary tumor growth of both the multidrug-resistant CT26 clone (Figure 2A) and the D2F2 clone (Figure 2B). To investigate whether we could further improve the efficacy of the vaccine, we combined it, first, with a vector encoding chemokine CCL21 (pCCL21) that had been previously shown to attract mature dendritic cells (23) as well as naive T cells (24), and second, with a vector encoding IL-2 (pIL2), a known inducer of $\mathrm{T}$ cell proliferation (25). However, the combination of the FAP vaccine with pCCL21 alone (Figure $2 \mathrm{~B}$ ) or with pIL2 and PCCL21 (Figure 2A) did not further improve its efficacy.

Vaccine against FAP reduces growth of established metastasis in a therapeutic setting. To determine whether the FAP vaccine could also inhibit tumor growth in a therapeutic setting of established experimental metastases, BALB/c mice were challenged i.v. with $1 \times 10^{5}$ CT26 colon carcinoma cells and then vaccinated on days 3 and 10 thereafter. We observed a significant decrease in tumor lung weights in pFap-vaccinated mice compared with the control group, which exhibited extensive metastases and showed severe signs of tumor cachexia 18 days after tumor cell inoculation (Figure 2C). Mice in the pFap group also showed a significant decrease in metastases on the lung surface as indicated by the corresponding metastasis scores (Figure 2C). 
A
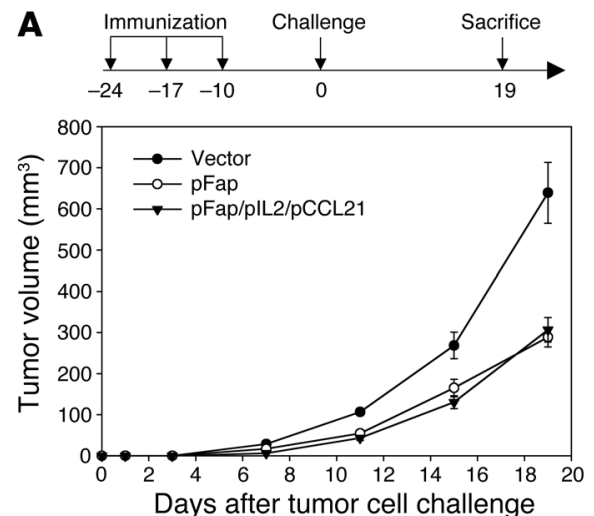

Days after tumor cell challenge
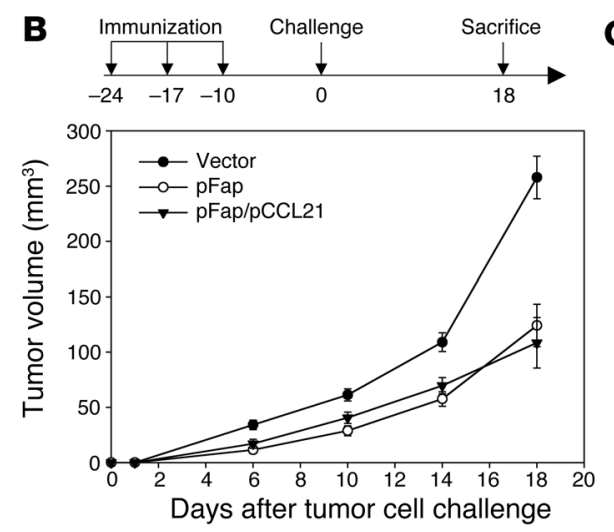
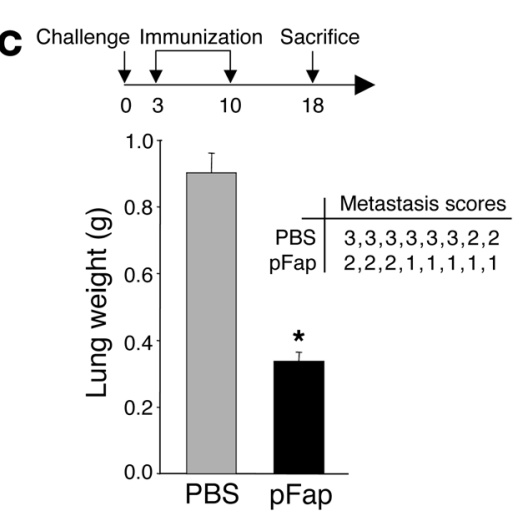

Figure 2

Effect of the FAP-based DNA vaccine on tumor growth. (A and B) Prophylactic setting. Ten days after the last of 3 vaccinations at 1 -week intervals, performed as described in Methods, BALB/c mice $(n=8)$ were challenged s.c. with a lethal dose of $3 \times 10^{4}$ CT26 cells $(\mathbf{A})$ or orthotopically with a lethal dose of $3 \times 10^{5} \mathrm{D} 2 \mathrm{~F} 2$ cells $(B)$. The mean \pm SEM of tumor growth of 8 mice is depicted. $P<0.01$. (C) Therapeutic setting. BALB/C mice $(n=8)$ were first injected i.v. with $10^{5}$ CT26 cells and then vaccinated after 3 and 10 days once pulmonary metastases were established. After 18 days, lungs were weighed (left panel, mean + SEM), examined for metastases, and scored by a visual evaluation (right panel) assessing the percentage of lung surface covered by fused metastases as follows: $0=0 \%, 1=<20 \%, 2=20-50 \%, 3=>50 \%$. ${ }^{\star} P<0.01$.

$C D 8^{+} T$ cells mediate an effective antitumor immune response. In order to determine which effector cell population accounts for the observed antitumor immune response, we challenged mice that were immunized i.v. 3 times at 1 -week intervals with $1 \times 10^{5}$ CT26 cells and depleted their respective effector cells during the effector phase with antibodies against $\mathrm{CD}^{+}{ }^{+}$or $\mathrm{CD}^{+} \mathrm{T}$ cells as well as NK cells (Figure 3A). We were able to establish a decisive role for $\mathrm{CD}^{+}$ $T$ cells, as we previously observed in a similar attempt with another DNA-based vaccine (26). Depletion of CD4 ${ }^{+}$cells and NK cells did not decrease the effectiveness of our vaccine.

To assess whether vaccination with our DNA-based FAP vaccine is able to break peripheral $\mathrm{T}$ cell tolerance against this self-antigen, we purified $\mathrm{CD}^{+} \mathrm{T}$ cells from animals vaccinated with vectors encoding the empty vector or FAP. We then stimulated these cells with $\gamma$-irradiated tumor target cells and incubated them with live tumor target cells that were transiently transfected with either GFP as a control or GFP plus pFap. As shown (Figure 3B), only $\mathrm{CD}^{+} \mathrm{T}$ cells purified from mice vaccinated with $\mathrm{pFap}$ were able to induce nuclear apoptosis as assessed by staining of pFap-transfected target cells with Hoechst 33342 dye. Splenocytes of immunized mice were also used in a conventional ${ }^{51} \mathrm{Cr}$-release assay. To this end, splenocytes from mice immunized with pFap and empty vector were incubated for 5 days with $\gamma$-irradiated A31 fibroblasts, which were retrovirally infected with pFap. Stimulated splenocytes were then incubated for 4 hours with labeled A31-pFap cells, and the percentage of lysis was calculated. Splenocytes from mice vaccinated with pFap were able to lyse significantly more fibroblasts than those from empty-vector controls at target-toeffector ratios of 1:100 and 1:25 (Figure 3C). Coincubation with anti-MHC class I antibodies abolished this effect (Figure 3D). To investigate whether tumors of pFap-vaccinated mice show an increase in $\mathrm{CD}^{+} \mathrm{T}$ cell infiltration, mice were immunized 3 times with either pFap or empty vector and then challenged with $3 \times 10^{4}$ CT26 tumor cells. After 3 weeks, tumors were harvested, and single-cell suspensions were stained for $\mathrm{CD}^{+}$and $\mathrm{CD}^{+}$cells and analyzed by FACS. Mice vaccinated with pFap showed a marked increase in $\mathrm{CD}^{+} \mathrm{CD}^{+}$cells in the tumor tissue when compared with the empty-vector controls (Figure 3E). Tumor sections were also stained with anti-CD8 ${ }^{+}$FITC and DAPI nuclear stain. By confocal microscopy, tumors of pFap-vaccinated mice showed a more marked infiltration with $\mathrm{CD}^{+}$cells than mice vaccinated with empty vector (Figure $3 \mathrm{~F}$ ). Taken together, these findings demonstrate that a DNA vaccine targeting FAP can overcome peripheral $T$ cell tolerance against the FAP self-antigen.

Suppression of collagen type I expression increases intratumoral uptake offluorescein, albumin, and 5-fluorouracil. Fibroblasts are the primary source of collagen type I, and it has been shown recently that expression of this molecule correlates inversely with intratumoral uptake of compounds of varying molecular weights (22-24). To evaluate whether this same mechanism is applicable to our FAP vaccine, we stained tumor sections of vaccinated mice with antibodies against FAP (Figure 4A, top) or against collagen type I (Figure $4 \mathrm{~A}$, bottom). We were able to detect a decrease in the expression of FAP as well as collagen type I in groups of mice vaccinated with pFap as compared with mice vaccinated only with the empty vector. Corresponding Western blots of these tumor extracts, stained with the same antibodies, revealed an $82.63 \% \pm 2.54 \%$ decrease in FAP expression (Figure 4B, top) and a $76.36 \% \pm 2.01 \%$ decrease in collagen type I expression (Figure 4B, middle).

We then injected mice with 1 of 3 structurally different compounds, fluorescein (376 Da) (Figure 4C), Evans blue albumin $(68,500 \mathrm{Da})$ (Figure 4D), or the chemotherapeutic agent 5-fluorouracil (130 Da) (Figure 4E), as described in Methods. Tumors of mice immunized with pFap DNA vaccines incorporated significantly more $(P<0.05)$ of these respective molecules than those of mice given the control vaccine.

Combination of vaccine and chemotherapy leads to tumor rejection. In order to evaluate the extent to which the findings depicted in Figure 4 suggest clinical relevance, we combined our DNA-based FAP vaccine with the chemotherapeutic drug doxorubicin, to which our D2F2 clone was partially sensitive (Figure 1C). In a manner similar to that of the experimental design shown in Figure 2B, we vaccinated $\mathrm{BALB} / \mathrm{c}$ mice $(n=8)$ with the empty control vector or the treatment vector $\mathrm{pFap}$ and then challenged these mice ortho- 

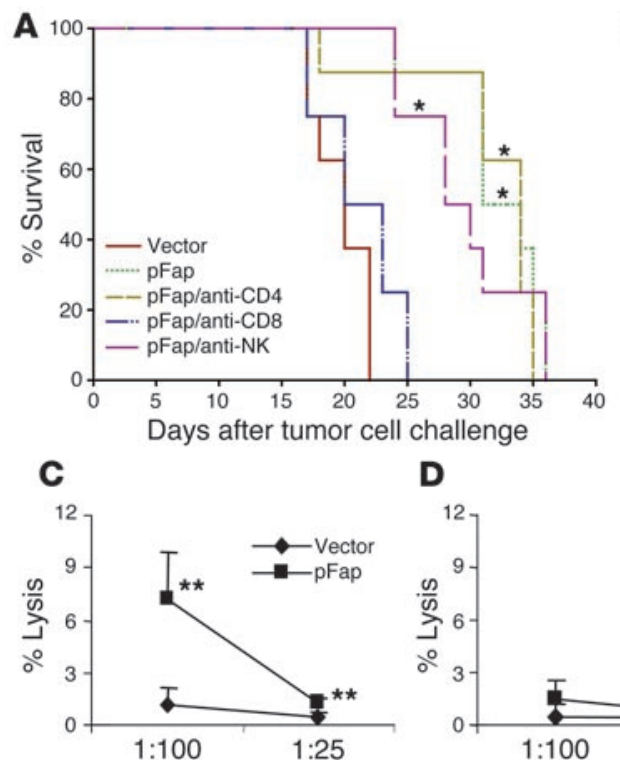

D
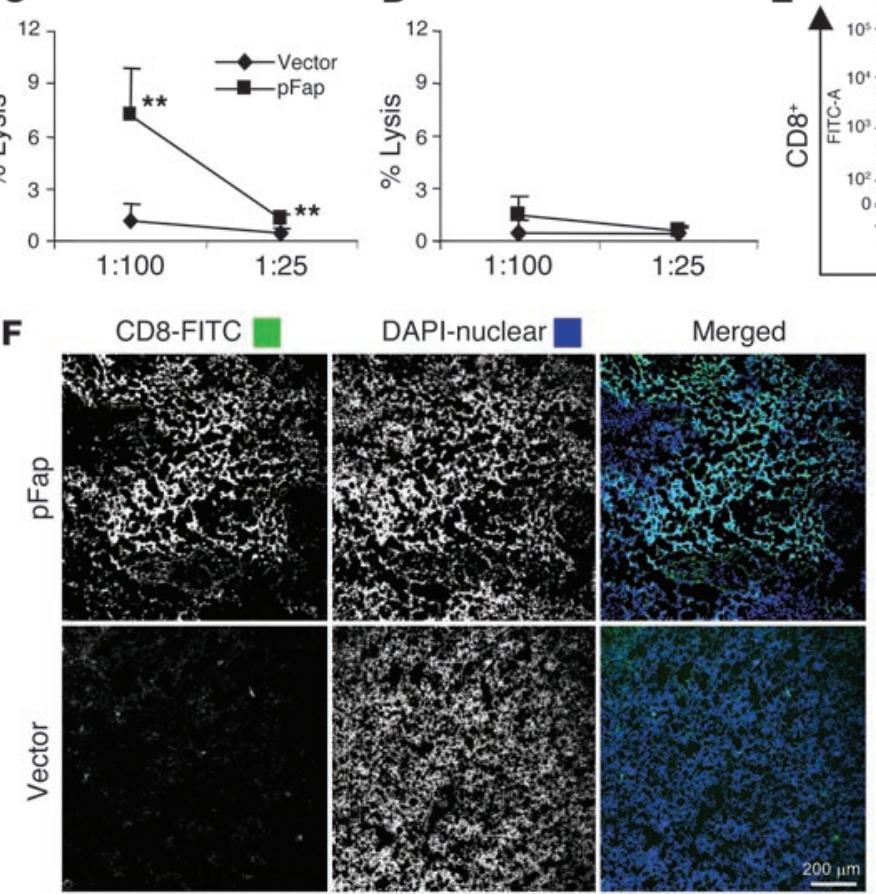

Figure 3
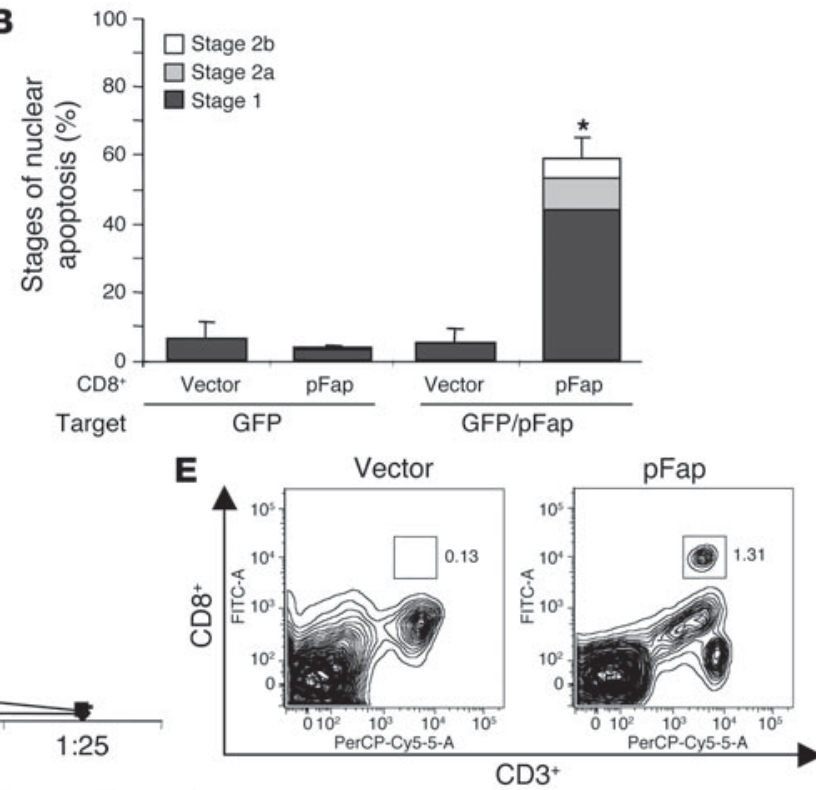

Antitumor effects of vaccine are mediated by CD8 ${ }^{+} \mathrm{T}$ cells. (A) The effect on lifespan of antibody-mediated depletion during the effector phase in vaccinated mice (3 times at 1-week intervals) challenged i.v. with $10^{5} \mathrm{CT} 26$ cells. (B) CD8 ${ }^{+} \mathrm{T}$ cells were purified from spleens of vaccinated mice, stimulated with $\gamma$-irradiated tumor target cells, and then incubated for 48 hours with GFP- or GFP/pFap-transfected CT26 cells. Nuclear apoptosis was assessed by staining with Hoechst 33342 dye as follows: nuclear apoptosis stage 0 , no apoptosis; stage 1, large-scale chromatin condensation; stage $2 \mathrm{a}$, chromatin fragmentation; stage $2 \mathrm{~b}$, apoptotic bodies. (C and D) Splenocytes from pFap- and empty vector-immunized mice $(n=3)$ were stimulated for 5 days with pFap-transfected A31 fibroblasts and then subjected to a ${ }^{51} \mathrm{Cr}$-release assay. (D) Effector and target cells were coincubated with anti-MHC class I antibodies (mean + SD). (E) FACS analysis of single-cell suspensions of CT26 tumors of vaccinated mice $(n=2)$ stained with anti-CD3 ${ }^{+}$PerCP-Cy5.5 and anti-CD8 ${ }^{+}$FITC antibodies. One of two experiments is depicted. $(F)$ Representative sections of CT26 tumors of pFap- and empty vector-vaccinated mice stained with anti-CD8 ${ }^{+}$FITC antibodies and DAPI nuclear stain. * Statistically significant compared with vector group, $P<0.01$. ${ }^{*}$ Statistically significant compared with empty vector, $P<0.05$.

topically with D2F2 breast carcinoma cells. We then treated these 2 groups of mice with either doxorubicin or PBS as a control 5, 10, and 15 days after tumor challenge. As shown, single treatments with either immunotherapy ( $\mathrm{pFap}$ ) or chemotherapy were able only to suppress but not to eradicate tumor growth (Figure 5A). In contrast, the group of mice treated with pFap and doxorubicin in combination revealed not only a marked inhibition of tumor growth, but also a complete tumor rejection in 4 of 8 mice (Figure $5 \mathrm{~A})$. In another combination therapy approach in a therapeutic setting, we first challenged BALB/c mice $(n=8)$ i.v. with D2F2 tumor cells. Once experimental metastasis had been established after 5 days, the mice were treated weekly with our FAP vaccine.
One day after each immunization, the mice were injected i.v. with doxorubicin. As a result of this combination treatment, the lifespan of these mice exceeded by more than 3 times that of control mice treated with doxorubicin or $\mathrm{pFap}$ alone, which died after only about 35-45 days (Figure 5B).

Vaccine against FAP increases intratumoral uptake of doxorubicin. To determine the doxorubicin concentration in the tumor tissue, we immunized mice $(n=4) 3$ times at 1 -week intervals, challenged them 7 days later with $5 \times 10^{5} \mathrm{D} 2 \mathrm{~F} 2$ tumor cells, and injected them 16 days later i.v. with doxorubicin to determine the intratumoral drug concentration with liquid chromatography and mass spectrometry (LC-MS). We found that, like tissues of mice injected 
A
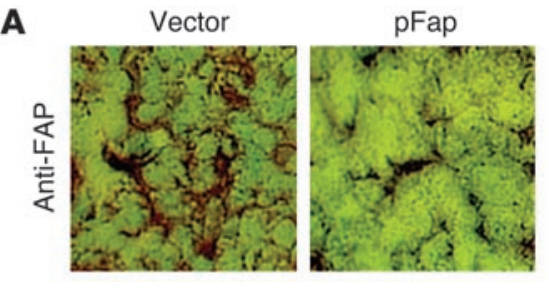

B
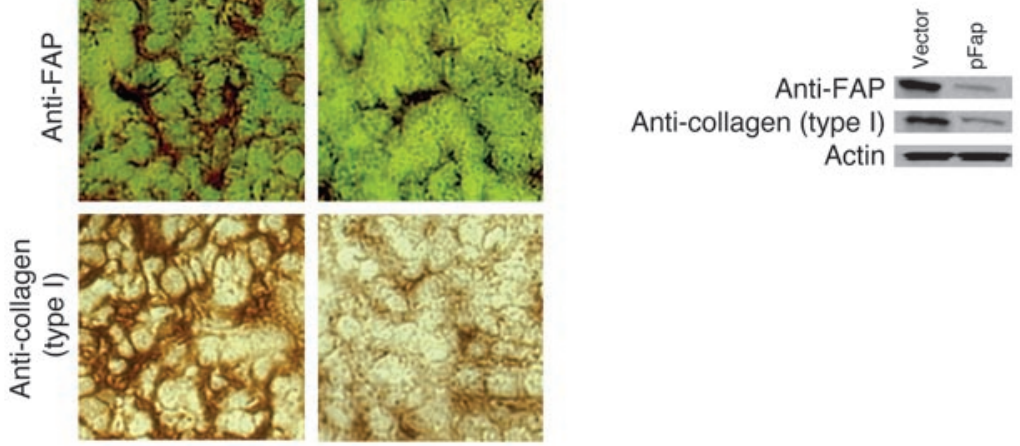

Actin

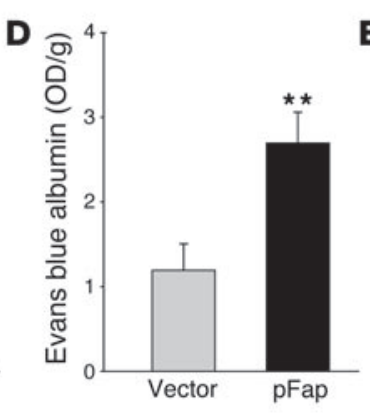

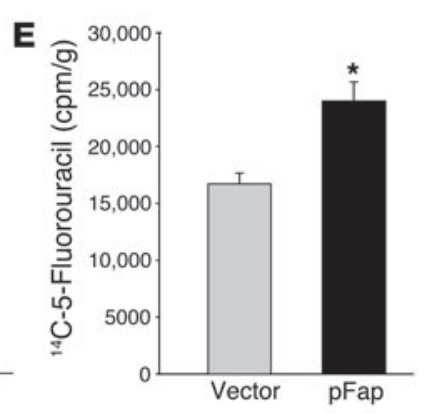

\section{Figure 4}

Expression of FAP and collagen type I and intratumoral uptake of fluorescein, albumin, and 5-fluorouracil. (A) Immunohistochemical analysis of FAP (top) and collagen type I (bottom) expression in s.c. CT26 tumors of vaccinated mice. (B) Immunoblots of FAP and collagen type I in s.c. CT26 tumors of vaccinated mice. (C-E) Bar graphs indicate mean + SEM of OD or scintillation measurements of homogenates of s.c. CT26 tumors from vaccinated BALB/c mice $(n=4)$, after i.p. injection of fluorescein, i.v. injection of Evans blue albumin, or i.v. injection of ${ }^{14} \mathrm{C}$-5-fluorouracil. ${ }^{\star} P<0.05,{ }^{* \star} P<0.01$.

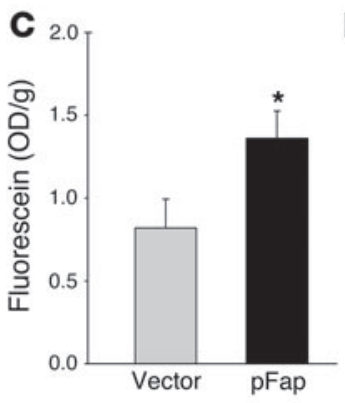

with fluorescein, albumin, and 5-fluorouracil (Figure 4, C-E), tissues of mice vaccinated with pFap showed a significant increase of doxorubicin in the tumor (Figure 5C).

The DNA vaccine against FAP does not impair wound healing or damage normal tissue. The fact that FAP is overexpressed during wound healing led us to evaluate whether a decrease in the number of cells expressing FAP could also lead to a prolongation of the time required to achieve complete wound healing. After inflicting a circular wound $3 \mathrm{~mm}$ in diameter on the backs of vaccinated BALB/c mice $(n=4)$, we did not observe any significant difference in wound healing between vaccinated and nonvaccinated mice (Figure 5D). Furthermore, histological assessment of these wounds by a mouse pathologist after different durations of time revealed no qualitative abnormalities in the wound healing process. To exclude any autoimmune reactions induced by our FAP vaccine, comprehensive histology of the following tissues and organs was performed: skin, brain, spinal cord, muscle, bone, synovium, heart, aorta, pulmonary artery, thymus, spleen, lymph nodes, bone marrow, parathyroid, adrenal gland, kidney, uterus, vagina, clitoral gland, tongue, liver, lungs, pancreas, stomach, small intestine, and colon. No discernible differences as compared with control mice were observed.

\section{Discussion}

We have demonstrated, for the first time to our knowledge, that the tumor-stromal antigen FAP can serve as a target for T cell-mediated cancer immunotherapy. This approach has several well-documented advantages over therapies directed against antigens solely expressed by tumor cells. First, stromal fibroblasts are genetically more stable than frequently mutating, heterogeneous tumor cell populations (2-4). As such, the expression of the target antigen remains more stable and serves as a more reliable target for immunotherapy. Second, antigen presentation by stromal fibroblasts to the $\mathrm{T}$ cell receptor complex is not impaired by downregulated
MHC class I antigen expression, as is frequently the case in human tumor cells $(27,28)$. Third, tumor cells often become increasingly resistant to $\mathrm{T}$ cell-mediated killing because of defects in apoptosis signaling pathways, upregulation of antiapoptotic proteins, or immunosuppressive effects against CTLs $(1,5)$. Fourth, targeting FAP, specifically overexpressed in over $90 \%$ of colon, breast, and lung carcinomas (18), allows for a therapeutic approach to treat a number of different malignancies, in contrast to therapies involving antigens that are expressed solely by specific tumor types.

Our experimental data demonstrate that we can break peripheral $\mathrm{T}$ cell tolerance against the FAP self-antigen by delivering its cDNA as an oral DNA vaccine with a doubly attenuated strain of Salmonella typhimurium to APCs in a secondary lymphoid organ, i.e., Peyer's patches of the small intestine. In prophylactic as well as therapeutic settings, the $\mathrm{CD}^{+} \mathrm{T}$ cell-mediated antitumor immune response induced by $\mathrm{pFap}$ vaccination inhibited tumor growth and significantly suppressed the dissemination of established pulmonary metastases in different murine tumor models, i.e., multidrug-resistant CT26 colon and D2F2 breast carcinoma. Additionally, we previously demonstrated that the administration of a DNA vaccine, using attenuated S. typhimurium as the vaccine carrier, can prolong antitumor effects up to 10 months and also achieve marked upregulation of T cell, NK cell, and dendritic cell activation markers $(26,29)$. Furthermore, in vivo depletion of $\mathrm{T}$ and NK cells in our current experiments indicates the involvement of $\mathrm{CD}^{+}$but not $\mathrm{CD}^{+} \mathrm{T}$ or NK cells. As shown here, the cytotoxic effect mediated by $\mathrm{CD}^{+} \mathrm{T}$ cells in vitro was specifically directed against target cells overexpressing the FAP antigen. We can also rule out nonspecific immune responses, since only $\mathrm{CD}^{+} \mathrm{T}$ cells from pFap-vaccinated mice induced in vitro cytotoxic lysis of tumor cells and fibroblasts overexpressing FAP. Moreover, tumors of pFap-vaccinated mice showed a more pronounced infiltration with $\mathrm{CD}^{+} \mathrm{T}$ cells than tumors of mice vaccinated with the empty- 
A
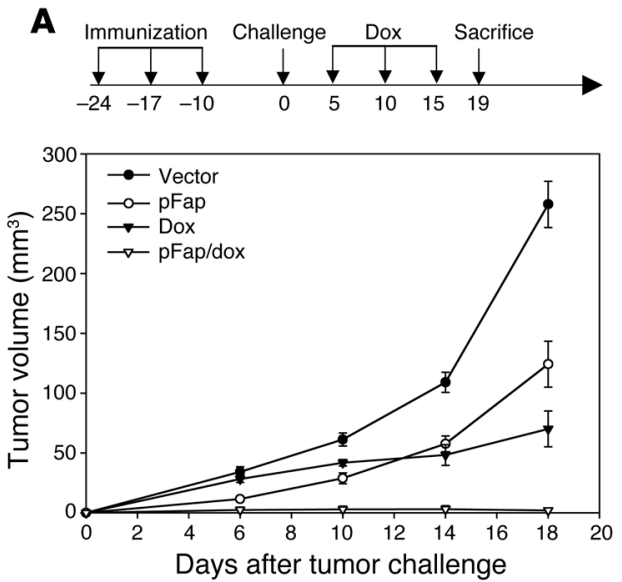

C

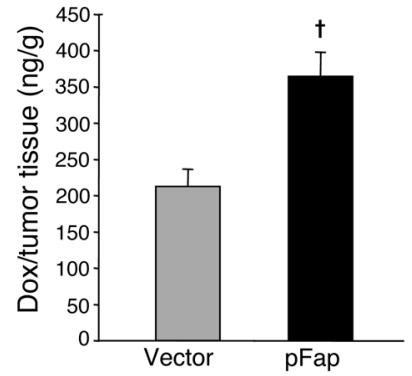

D
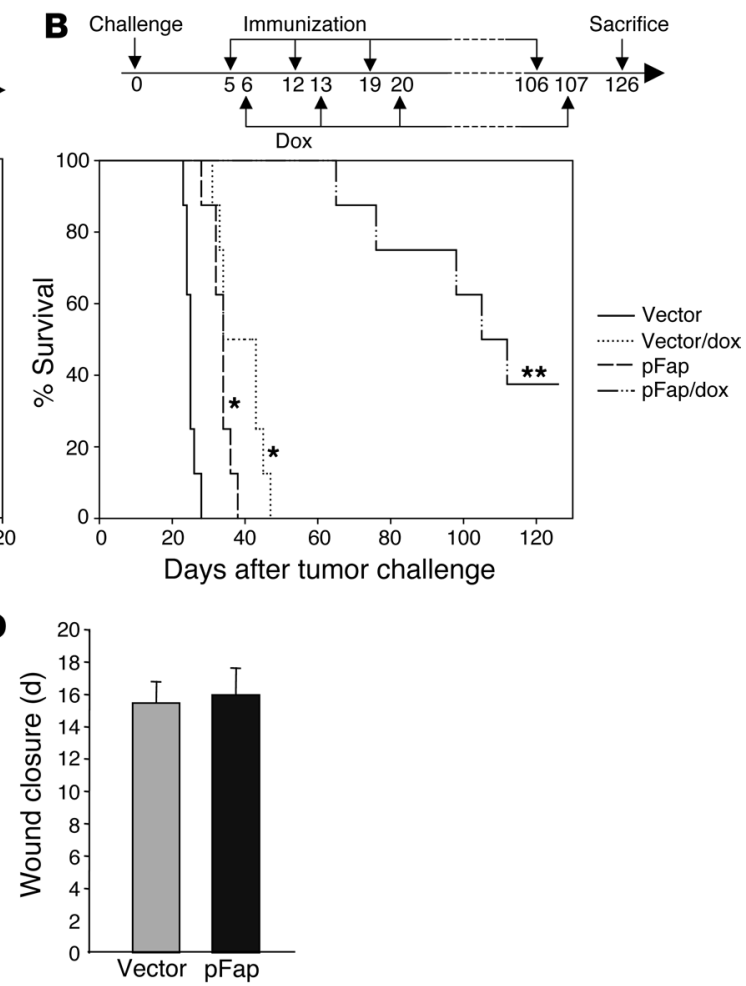

Figure 5

Antitumor and antimetastatic effects of combined bio- and chemotherapy and side effects. (A) Prophylactic setting. Ten days after the last of 3 vaccinations at 1 -week intervals with empty vector, PBS, or $\mathrm{pFap}$, BALB/c mice $(n=8$, mean $\pm \mathrm{SEM})$ were challenged orthotopically with $3 \times 10^{5}$ D2F2 cells. After 5,10 , and 15 days, indicated mice were treated with doxorubicin (dox). (B) Therapeutic setting. Five days after i.v. injection of $10^{5} \mathrm{D} 2 \mathrm{~F} 2$ tumor cells, BALB/c mice $(n=8)$ were treated weekly with pFap or empty vector. One day after each immunization, mice were treated with doxorubicin i.v. as indicated (*statistically significant compared with vector group, $P<0.0001$; ${ }^{* *}$ statistically significant compared with vector, vector/dox, and pFap groups, $P<0.0001)$. (C) Intratumoral doxorubicin concentration. Vaccinated BALB/c mice $(n=4)$ were challenged s.c. with $5 \times 10^{5}$ D2F2 cells, and after 16 days the doxorubicin concentration in pooled tumor lysates was determined by liquid chromatography and mass spectrometry (LC-MS) (representative of 2 experiments, mean $\left.+\mathrm{SD},{ }^{\dagger} P<0.001\right)$. (D) Circular wounds 3 mm in diameter were inflicted on the upper backs of vaccinated mice $(n=4)$, and the average time until complete wound closure was measured (mean + SD).

vector control. Even though FAP can also be found transiently overexpressed during wound healing, vaccination against FAP did not impair wound healing, nor did it cause any discernible autoimmune reactions, in the 26 tissues and organs examined histologically. Functional redundancy of proteins with overlapping function may account for this observation (30).

We also tested 3 different effector molecules as adjuvants in hopes of improving the efficacy of our vaccine: IL-2, an inducer of $\mathrm{T}$ cell proliferation (31); CCL21, a chemokine that chemoattracts mature dendritic cells (32) and naive T cells (33); and CD40LT, a known inducer of dendritic cell maturation (34) (data not shown). Coadministration of all 3 of these effector molecules failed to improve the efficacy of the vaccine. This indicates that the CTL response induced against tumor-stromal fibroblasts is sufficient to inhibit the growth of different tumors, but not adequate to reject them as a single entity. This may be due to the fact that tumor cells and tumor-associated macrophages can elicit redundancy by synthesizing a variety of tumor-promoting proteins, such as $\operatorname{VEGF}(35,36)$, TGF- $\beta_{1}(37,38)$, and IL-10 $(38,39)$, the serum concentrations of which we were unable to lower with our vaccine (data not shown).

The rationale for combining cancer vaccines with chemotherapy has been extensively documented and reviewed elsewhere (40). In addition to the immunostimulatory potential of various chemotherapeutics, such as doxorubicin, paclitaxel, and cyclophosphamide, which do not cause bone marrow suppression when administered at low doses, the combination of our FAP vaccine with such drugs offers an additional advantage, specifically the downregulation of collagen type I expression, and consequently, increased intratumoral uptake of different chemotherapeutic agents. We demonstrated this with 3 structurally unrelated molecules: the low-molecular-weight dye fluorescein $(376 \mathrm{Da})$, the high-molecular-weight compound Evans blue albumin $(68,500 \mathrm{Da})$, and the chemotherapeutic agent 5 -fluorouracil (130 Da). Seemingly, the changes that occur in the pFap-vaccinated animals facilitate both processes responsible for intratumoral transport, i.e., diffusion for small molecules as well as convection for larger molecules (41-44). These findings support a novel rationale for improving chemotherapy by combining it with immunotherapy. Indeed, we demonstrated that the combination of a DNA vaccine encoding FAP plus doxorubicin can induce complete rejection of orthotopically grown breast tumors in $50 \%$ of mice as well as a more than 3 -fold prolongation of the lifespan of mice with experimentally induced breast cancer metastases, a result that was not achieved when either of these treatments was applied alone. Furthermore, we demonstrated that the amount of doxorubicin in the tumor tissue 
of pFap-vaccinated mice is significantly increased when compared with that in control mice vaccinated with empty vector.

Taken together, our findings demonstrate, for the first time to our knowledge, the efficacy of an oral DNA vaccine in eliciting an immune response mediated by $\mathrm{CD}^{+} \mathrm{T}$ cells against the tumorstromal antigen FAP. More importantly, our DNA-based vaccine against FAP, when combined with doxorubicin, was able to inhibit tumor growth and lead to complete tumor rejection in $50 \%$ of mice. In addition we observed a more than 3 -fold prolonged lifespan in a breast cancer model of experimental metastases, which was at least partly due to increased drug uptake at the tumor site. It is anticipated that this and other studies that involve combinations of immuno- and chemotherapies may soon lead to new approaches in clinical practice and improvements in currently available treatments for cancer.

\section{Methods}

Animals, bacterial strains, and cell lines. Female BALB/c mice, 6-8 weeks of age, were obtained from The Scripps Research Institute Rodent Breeding Facility. All animal experiments were performed according to the NIH Guide for the Care and Use of Laboratory Animals and approved by The Scripps Research Institute Animal Care Committee. The doubly attenuated S. typhimurium strain RE88 ( $\mathrm{rroA}^{-} \mathrm{dam}^{-}$) was provided by Remedyne Corp. Murine D2F2 breast carcinoma cells were a gift from Wei-Zen Wei (Department of Immunology, Karmanos Cancer Institute, Detroit, Michigan, USA), and CT26 colon carcinoma cells were originally obtained from ATCC and then cultivated over several months to obtain chemoresistant subclones.

Vector and cell-line characterization. The cDNAs encoding murine FAP (provided by J.D. Cheng, Department of Medical Oncology, Fox Chase Cancer Center, Philadelphia, Pennsylvania, USA), IL-2 (ATCC), and CCL21 (InvivoGen) were subcloned into the pcDNA3.1/V5-His-TOPO vector (Invitrogen). For transient transfections, CT26 or D2F2 cells were electroporated as described previously (45). Protein expression of pFap (pCCL21 and pIL2 not shown) was demonstrated by Western blotting with a polyclonal rabbit anti-murine FAP antibody provided by J.D. Cheng. Murine CT26 and D2F2 carcinoma cells were cultured in the presence of $1 \%$ DMSO, etoposide, 5-fluorouracil, doxorubicin, vinblastine, or paclitaxel (Sigma-Aldrich) at the concentrations indicated. After 48 hours, apoptotic nuclei were counted after incubation with the DNA-specific Hoechst 33342 dye ( $2 \mu \mathrm{M}$; Invitrogen).

Oral immunization, tumor cell challenge, and treatment with doxorubicin and antibodies against effector cells. For experiments in a prophylactic setting, $\mathrm{BALB} / \mathrm{c}$ mice $(n=8)$ were immunized 3 times at 1 -week intervals by gavage with $100 \mu \mathrm{l}$ PBS containing $10^{9}$ S. typhimurium $\left(\right.$ AroA $^{-}$dam $\left.{ }^{-}\right)$transformed with the plasmid vectors indicated, as described previously (26). Animals were challenged 10 days later by s.c. injection of $3 \times 10^{4} \mathrm{CT} 26$ colon carcinoma cells in the left front flank or by orthotopic injection of $3 \times 10^{5}$ D2F2 breast cancer cells in the no. 4 mammary fat pad. Tumor volume was measured in 2 dimensions and calculated as length $/ 2 \times$ width $^{2}$. In the experimental therapeutic setting, the initial i.v. tumor cell inoculation with $1 \times 10^{5} \mathrm{CT} 26$ colon carcinoma cells was followed 3 and 10 days later by oral vaccinations. After 18 days, lungs were weighed (normal lung weight $=0.2 \mathrm{~g}$ ), and pulmonary tumor metastases were examined and scored by visual evaluation assessing the percentage of lung surface covered by fused metastases as follows: $0=0 \%, 1=<20 \%, 2=20-50 \%$, $3=>50 \%$. Treatment with doxorubicin was performed in experimental groups of mice $(n=8)$ with $10 \mathrm{mg} / \mathrm{kg}$ doxorubicin (Sigma-Aldrich) administered i.v. 5, 10, and 15 days after tumor cell challenge in the prophylactic setting. In the therapeutic setting, mice were immunized weekly starting on day 5 after tumor cell challenge; doxorubicin was given weekly always 1 day after the immunization as indicated.
For the depletion of $\mathrm{CD}^{+}$and $\mathrm{CD}^{+} \mathrm{T}$ cells or $\mathrm{NK}$ cell subpopulations, antibodies $(500 \mu \mathrm{g})$ directed against either CD4 (clone GK 1.5) or CD8 (clone 2.43), both from National Cell Culture Center (Minneapolis, Minnesota, USA), or anti-asialo-GM1 antibody (Wako) were injected i.p. every 7 days starting 1 day before tumor cell challenge.

Cytotoxicity of $C D 8^{+} T$ cells. Ten days after the last of 3 immunizations at 1-week intervals, splenocytes from vaccinated groups of $\mathrm{BALB} / \mathrm{c}$ mice $(n=4)$ were collected. Using CD8a MicroBeads (Miltenyi Biotec), CD8 ${ }^{+}$ cells were purified according to the manufacturer's protocol. The cells were then stimulated in a 5-day coculture with $\gamma$-irradiated (1,000 Gy, 45 minutes) CT26 cells transiently transfected with either empty vector or pFap. Thereafter, $\mathrm{CD}^{+} \mathrm{T}$ cells were cocultivated with CT26 carcinoma cells (effector-to-target ratio $=100: 1$ ) transiently transfected with either GFP (pEGFP; Clontech) as a control or GFP plus pFap. After 48 hours, nuclear apoptosis was assessed as described previously (45) with the DNA-specific Hoechst 33342 dye $(2 \mu \mathrm{M})$. For the ${ }^{51} \mathrm{Cr}$-release assay, BALB/c mice $(n=3)$ were immunized 4 times at weekly intervals. Splenocytes were harvested 13 days after the last immunization and incubated for 5 days with $\gamma$-irradiated (1,000 Gy, 45 minutes) A31 fibroblasts (ATCC), which were retrovirally infected with pFap. Stimulated splenocytes were then incubated for 4 hours with labeled A31-pFap, and the percentage of lysis was calculated. Cells were also coincubated with anti-MHC class I antibodies (BD Biosciences) at a concentration of $10 \mu \mathrm{g} / \mathrm{ml}$.

Immunohistochemistry and T cell infiltration. Cryosections $(8 \mu \mathrm{m})$ of tumors were fixed in acetone. After incubation with the primary antibody, either a polyclonal rabbit anti-murine FAP antibody (provided by J.D. Cheng) or a polyclonal rabbit anti-murine collagen type I antibody (Chemicon International), the sections were immunostained according to the manufacturer's protocol (Universal LSAB 2 Single Reagents, Peroxidase; Dako). For focal microscopy, fixated cryosections were stained with anti-murine CD8 antibody, biotinylated anti-rat Ig secondary antibody, and FITC-labeled streptavidin (BD Biosciences) and costained with DAPI (Sigma-Aldrich). A Bio-Rad (Zeiss) Radiance 2100 Rainbow laser scanning confocal microscope was used to obtain images, which were processed using Zeiss LSM Image Examiner software. For FACS analysis of T cell infiltration, BALB/c mice $(n=6)$ were immunized 3 times at weekly intervals with either $\mathrm{pFap}$ or empty vector. One week after the last immunization, animals were challenged s.c. with $3 \times 10^{5}$ CT26 tumor cells. Tumors were harvested 3 weeks later, and single-cell suspensions were prepared by incubation of sliced tumor tissue for 45 minutes in medium supplemented with collagenase type I (125 U/ml; Invitrogen). After filtering, cells of 2 mice were pooled, stained with anti-CD3 ${ }^{+}$PerCpCy5.5 and anti-CD8 ${ }^{+}$FITC (BD Biosciences), and analyzed by FACS.

Intratumoral uptake of fluorescein, Evans blue albumin, ${ }^{14} \mathrm{C}-5$-fluorouracil, and doxorubicin. Ten days after the last of 3 vaccinations at 1 -week intervals, mice were challenged s.c. with $3 \times 10^{4}$ CT2 6 cells in the left front flank. Nineteen days later, mice were given injections of $1 \%$ fluorescein sodium (Sigma-Aldrich) i.p. at $12 \mu \mathrm{l} / \mathrm{g}$ of body weight, $100 \mu \mathrm{l}$ Evans blue albumin (Sigma-Aldrich) i.v., or $2.5 \mu \mathrm{Ci}{ }^{14} \mathrm{C}-5$-fluorouracil (Sigma-Aldrich) i.v. After 5 minutes, 30 minutes, or 1 hour, respectively, mice were killed to determine absorption or scintillation of the supernatants of tumor homogenates at $490 \mathrm{~nm}$, at $612 \mathrm{~nm}$, or in a gamma counter, respectively. For the determination of intratumoral doxorubicin uptake, BALB/c mice $(n=4)$ were challenged s.c. with $5 \times 10^{5} \mathrm{D} 2 \mathrm{~F} 2$ cells in the right flank. Doxorubicin was injected i.v. 16 days later $(10 \mathrm{mg} / \mathrm{kg})$ and tumors harvested 45 minutes thereafter. Samples were prepared as described previously (46) and measured against the internal standard daunorubicin using a ZORBAX Eclipse XDB-C8 column on an 1100 series liquid chromatography-mass spectrometry (LC-MS) system (Agilent Technologies).

Evaluation of side effects. In order to determine any detrimental effects on wound healing, wounding was performed as previously described 
$(47,48)$. We inflicted a circular wound $3 \mathrm{~mm}$ in diameter with a dermal punch (Miltex Inc.) on the upper backs of BALB/c mice $(n=4), 10$ days after the last of 3 immunizations at 1 -week intervals, and measured the time until complete wound closure. For histological analysis, we inflicted wounds on vaccinated mice 7, 14, and 21 days before examination. Skin biopsies and 26 organs and tissues were examined by a mouse pathologist.

Statistics. The statistical significance of differential findings between experimental groups and controls was determined by 2-tailed Student's $t$ test. The significance of metastasis scores was determined by Mann-Whitney $U$ test. The significance of survival data was determined by the log-rank test. Findings were regarded as significant if $P$ values were less than 0.05 .

\section{Acknowledgments}

We thank Wei-Zen Wei, who kindly supplied us with the murine D2F2 breast carcinoma cells, and J.D. Cheng, who kindly pro- vided the cDNA for murine FAP as well as the polyclonal antibody against the protein. This study was supported by NIH grant CA83856 (to R.A. Reisfeld), grant SFP1330 from the EMD Lexigen Research Center (to R.A. Reisfeld), and Department of Defense grant BC031079 (to R.A. Reisfeld).

Received for publication August 9, 2005, and accepted in revised form May 2, 2006.

Address correspondence to: R.A. Reisfeld, The Scripps Research Institute, Department of Immunology, 10550 North Torrey Pines Road, La Jolla, California 92037, USA. Phone: (858) 784-8105; Fax: (858) 784-2708; E-mail: reisfeld@scripps.edu.

Markus Loeffler and Jörg A. Krüger contributed equally to this work.
1. Marincola, F.M., Wang, E., Herlyn, M., Seliger, B., and Ferrone, S. 2003. Tumors as elusive targets of T-cell-based active immunotherapy. Trends Immunol. 24:335-342.

2. Cohen, S., Regev, A., and Lavi, S. 1997. Small polydispersed circular DNA (spcDNA) in human cells: association with genomic instability. Oncogene. 14:977-985.

3. Boehm, T., Folkman, J., Browder, T., and O'Reilly, M.S. 1997. Antiangiogenic therapy of experimental cancer does not induce acquired drug resistance. Nature. 390:404-407.

4. Vitale, M., et al. 1998. HLA class I antigen and transporter associated with antigen processing (TAP1 and TAP2) down-regulation in high-grade primary breast carcinoma lesions. Cancer Res. 58:737-742.

5. Reed, J.C. 2003. Apoptosis-targeted therapies for cancer. Cancer Cell. 3:17-22.

6. Liotta, L.A., and Kohn, E.C. 2001. The microenvironment of the tumour-host interface. Nature. 411:375-379.

7. Pupa, S.M., Menard, S., Forti, S., and Tagliabue, E. 2002. New insights into the role of extracellular matrix during tumor onset and progression. J. Cell. Physiol. 192:259-267.

8. Wiseman, B.S., and Werb, Z. 2002. Stromal effects on mammary gland development and breast cancer. Science. 296:1046-1049.

9. Singh, S., Ross, S.R., Acena, M., Rowley, D.A., and Schreiber, H. 1992. Stroma is critical for preventing or permitting immunological destruction of antigenic cancer cells. J. Exp. Med. 175:139-146.

10. Schuler, T., Kornig, S., and Blankenstein, T. 2003. Tumor rejection by modulation of tumor stromal fibroblasts. J. Exp. Med. 198:1487-1493.

11. Ibe, S., Qin, Z., Schuler, T., Preiss, S., and Blankenstein, T. 2001. Tumor rejection by disturbing tumor stroma cell interactions. J. Exp. Med. 194:1549-1559.

12. Bhowmick, N.A., et al. 2004. TGF-beta signaling in fibroblasts modulates the oncogenic potential of adjacent epithelia. Science. 303:848-851.

13. Gabrilovich, D.I., et al. 1996. Production of vascular endothelial growth factor by human tumors inhibits the functional maturation of dendritic cells. Nat. Med. 2:1096-1103.

14. Elgert, K.D., Alleva, D.G., and Mullins, D.W. 1998. Tumor-induced immune dysfunction: the macrophage connection. J. Lenkoc. Biol. 64:275-290.

15. O'Connor, D.S., et al. 2000. Control of apoptosis during angiogenesis by survivin expression in endothelial cells. Am. J. Pathol. 156:393-398.

16. Muraoka, R.S., et al. 2002. Blockade of TGF- $\beta$ inhibits mammary tumor cell viability, migration, and metastases. J. Clin. Invest. 109:1551-1559. doi:10.1172/JCI200215234.

17. Yang, Y.-A., et al. 2002. Lifetime exposure to a soluble TGF- $\beta$ antagonist protects mice against metas- tasis without adverse side effects. J. Clin. Invest. 109:1607-1615. doi:10.1172/JCI200215333.

18. Scanlan, M.J., et al. 1994. Molecular cloning of fibroblast activation protein alpha, a member of the serine protease family selectively expressed in stromal fibroblasts of epithelial cancers. Proc. Natl. Acad. Sci. U. S. A. 91:5657-5661.

19. Park, J.E., et al. 1999. Fibroblast activation protein, a dual specificity serine protease expressed in reactive human tumor stromal fibroblasts. J. Biol. Chem. 274:36505-36512.

20. Cheng, J.D., et al. 2002. Promotion of tumor growth by murine fibroblast activation protein, a serine protease, in an animal model. Cancer Res. 62:4767-4772.

21. Lee, J., Fassnacht, M., Nair, S., Boczkowski, D., and Gilboa, E. 2005. Tumor immunotherapy targeting fibroblast activation protein, a product expressed in tumor-associated fibroblasts. Cancer Res. 65:11156-11163.

22. Netti, P.A., Berk, D.A., Swartz, M.A., Grodzinsky, A.J., and Jain, R.K. 2000. Role of extracellular matrix assembly in interstitial transport in solid tumors. Cancer Res. 60:2497-2503.

23. Boucher, Y., Brekken, C., Netti, P.A., Baxter, L.T., and Jain, R.K. 1998. Intratumoral infusion of fluid: estimation of hydraulic conductivity and implications for the delivery of therapeutic agents. $\mathrm{Br}$. J. Cancer. 78:1442-1448.

24. Heldin, C.H., Rubin, K., Pietras, K., and Ostman, A. 2004. High interstitial fluid pressure: an obstacle in cancer therapy. Nat. Rev. Cancer. 4:806-813.

25. Gabbiani, G. 2003. The myofibroblast in wound healing and fibrocontractive diseases. J. Pathol. 200:500-503.

26. Niethammer, A.G., et al. 2002. A DNA vaccine against VEGF receptor 2 prevents effective angiogenesis and inhibits tumor growth. Nat. Med. 8:1369-1375.

27. Muris, J.J., et al. 2004. Prognostic significance of activated cytotoxic T-lymphocytes in primary nodal diffuse large B-cell lymphomas. Lenkemia. 18:589-596.

28. Johnsen, A., France, J., Sy, M.S., and Harding, C.V. 1998. Down-regulation of the transporter for antigen presentation, proteasome subunits, and class I major histocompatibility complex in tumor cell lines. Cancer Res. 58:3660-3667.

29. Luo, Y., et al. 2003. Transcription factor Fos-related antigen 1 is an effective target for a breast cancer vaccine. Proc. Natl. Acad. Sci. U. S. A. 100:8850-8855.

30. Niedermeyer, J., et al. 2000. Targeted disruption of mouse fibroblast activation protein. Mol. Cell. Biol. 20:1089-1094.

31. Lotze, M.T. 1992. T-cell growth factors and the treatment of patients with cancer. Clin. Immunol. Immunopathol. 62:S47-S54.

32. Dieu-Nosjean, M.C., Vicari, A., Lebecque, S., and Caux, C. 1999. Regulation of dendritic cell traf- ficking: a process that involves the participation of selective chemokines. J. Leukoc. Biol. 66:252-262.

33. Kim, C.H., and Broxmeyer, H.E. 1999. Chemokines: signal lamps for trafficking of $\mathrm{T}$ and $\mathrm{B}$ cells for development and effector function. J. Leukoc. Biol. 65:6-15.

34. O'Sullivan, B., and Thomas, R. 2003. CD40 and dendritic cell function. Crit. Rev. Immunol. 23:83-107.

35. Brekken, R.A., and Thorpe, P.E. 2001. Vascular endothelial growth factor and vascular targeting of solid tumors. Anticancer Res. 21:4221-4229.

36. Khorana, A.A., Ryan, C.K., Cox, C., Eberly, S., and Sahasrabudhe, D.M. 2003. Vascular endothelial growth factor, CD68, and epidermal growth factor receptor expression and survival in patients with Stage II and Stage III colon carcinoma: a role for the host response in prognosis. Cancer. 97:960-968.

37. Kataki, A., et al. 2002. Tumor infiltrating lymphocytes and macrophages have a potential dual role in lung cancer by supporting both host-defense and tumor progression. J. Lab. Clin. Med. 140:320-328.

38. Platsoucas, C.D., et al. 2003. Immune responses to human tumors: development of tumor vaccines. Anticancer Res. 23:1969-1996.

39. Sica, A., et al. 2000. Autocrine production of IL-10 mediates defective IL-12 production and NF-kappa $B$ activation in tumor-associated macrophages. J. Immunol. 164:762-767.

40. Emens, L.A., Machiels, J.P., Reilly, R.T., and Jaffee, E.M. 2001. Chemotherapy: friend or foe to cancer vaccines? Curr. Opin. Mol. Ther. 3:77-84.

41. Leonard, E.F., and Jorgensen, S.B. 1974. The analysis of convection and diffusion in capillary beds. Annu. Rev. Biophys. Bioeng. 3:293-339.

42. Jain, R.K. 1987. Transport of molecules in the tumor interstitium: a review. Cancer Res. 47:3039-3051.

43. Jain, R.K. 1990. Physiological barriers to delivery of monoclonal antibodies and other macromolecules in tumors. Cancer Res. 50(Suppl. 3):814s-819s.

44. Baxter, L.T., and Jain, R.K. 1989. Transport of fluid and macromolecules in tumors. I. Role of interstitial pressure and convection. Microvasc. Res. 37:77-104.

45. Loeffler, M., et al. 2001. Dominant cell death induction by extramitochondrially targeted apoptosis-inducing factor. FASEB J. 15:758-767.

46. Munck, J.N., et al. 1993. Pharmacokinetic and pharmacodynamic advantages of pirarubicin over adriamycin after intraarterial hepatic administration in the rabbit VX2 tumor model. Cancer Res. 53:1550-1554.

47. Kaesler, S., Regenbogen, J., Durka, S., Goppelt, A., and Werner, S. 2002. The healing skin wound: a novel site of action of the chemokine C10. Cytokine. 17:157-163.

48. Werner, S., et al. 1992. Large induction of keratinocyte growth factor expression in the dermis during wound healing. Proc. Natl. Acad. Sci. U. S. A. 89:6896-6900. 